\title{
Atomistically Derived Cohesive Behavior of Interphases in Carbon Fiber Reinforced CNT Nanocomposites
}

\author{
Nithya Subramanian ${ }^{1}$, Ashwin Rai ${ }^{1}$, and Aditi Chattopadhyay ${ }^{1}$. \\ ${ }^{1}$ Arizona State University, 551 E. Tyler Mall, Tempe, AZ, 85287
}

\begin{abstract}
The carbon fiber/polymer matrix interphase region plays an important role in failure initiation and accurate modeling techniques are integral to study the effects of this complex region on the composite response. In composites infused with nanoparticles such as carbon nanotubes (CNT), the interphase region is more complex due to the presence of multiple constituents and their interactions with each other. An atomistic methodology to simulate the constituent interphases in carbon fiber reinforced CNT/epoxy nanocomposites is presented in this paper. The interphase model consisting of voids in multiple graphene layers enable the simulation of physical entanglement between the polymer matrix and the irregular carbon fiber surface. The voids in graphene layers are generated by removing carbon atoms and hydrogenating the end carbon atoms, which better represent the roughness of the carbon fiber surface. The epoxy curing studies and the response of fiber/matrix interphase under mechanical loading are investigated through molecular dynamic (MD) simulations with appropriate classical/harmonic and bond order-based force fields. Furthermore, the atomistic forcedisplacement behavior is also extracted to formulate a traction-separation law for interface cohesive zone models. The cohesive behavior determined from molecular models is parameterized in equations that can be integrated with an atomistically informed multiscale modeling framework.
\end{abstract}

\section{Introduction}

Recent research on CNT-enhanced nanocomposites suggests improved multifunctional capabilities including increased electrical and thermal conductivities, fracture toughness, fatigue threshold energy release rate, resistance to impact damage, and delay in the onset of delamination [1-4]. Furthermore, CNT nanocomposites have exhibited improved bonding and

*Corresponding Author: Tel: (480) 965 9342, Email: aditi@asu.edu (Aditi Chattopadhyay) 
adhesive properties at metal-composite interfaces, which leads to tougher carbon fiber-metal hybrid structures with stronger interfaces [5]. However, nano-engineered composites have multiple interfaces that play a significant role in the load transfer mechanism between constituent phases. Experimental investigations at nanoscale interfaces are challenging due to the limitations in direct measurement techniques, test specimen size and preparation, and uncertainty in the data from indirect measurements [6,7]. Hence, advanced predictive tools must be developed that capture scale-specific phenomena sufficiently and reasonably in order to predict the impact of the nanomaterial on the overall composite performance.

Computational methods using atomistic simulations have been used to understand the physical and chemical parameters that affect the mechanical response of the interphase [8,9] in nanocomposites. There is limited understanding of load transfer efficiency at the polymerreinforcement and the polymer-fiber interphases, which is strongly influenced by irregularities such as misalignment and defects in nano-reinforcements, surface roughness and matrix voids. Interfacial bonding in the interphase region between the embedded CNT and its surrounding polymer also plays a critical role in damage initiation at lower length scales. Although micromechanics models for the CNT-polymer interface have been developed [9,10], the effect of the interfacial covalent bonds on the system response cannot be characterized accurately using these models. Odegard et al. [11,12] developed an effective CNT-polymer interphase model based on coarse grained atomistic simulations for functionalized interfaces. This coarse-grained model enabled the simulation of continuum level time scales; however, load transfer mechanism at the CNT-polymer interface was not addressed. The cohesive zone modeling (CZM) approach has been explored in recent literature for modeling various interface zones, such as adhesive debonding [13], delamination in composites [14], fracture in asphalt concrete [15] and fatigue crack growth [16]. The CZM method presents several advantages in the modeling of interface debonding such as the accurate prediction of debonding in pristine interfaces, elimination of singularity issues and the capability to include plasticity based debonding behavior [17]. The validity of the CZM approach for any application relies critically on the traction-separation behavior of the interface. The estimation of the traction-separation behavior requires characterization of the atomistic process of debonding. The challenges posed by the resolution of existing experimental techniques lead most researchers to utilize an inverse approach to formulate an empirical traction-separation behavior and phenomenologically tuning the 
parameters by comparing with macroscale experimental observations. However, such approaches fail to capture the physics behind interfacial damage nucleation and evolution. Recently, Chandra [18] applied the CZM approach to model debonding of the CNT/polymer interphase by estimating the traction-separation behavior using atomistic models of nanoscale fracture formation. Jiang and coworkers formulated an interfacial cohesive law for the CNT/polymer interphase based on micromechanics approximations accounting for van der Waals interactions between the cylindrical nanotube and the polymer phase [10]. Although this framework provided a physically meaningful traction-separation law, the covalent bonds and other chemical interactions that affect mechanical response were not addressed. Shin and Dandekar used MD simulations to develop a cohesive zone law for describing the mechanics at the aluminum-silicon carbide interface; they implemented the embedded atom model potential, which is capable of capturing bond formation and dissociation in metals. However, since their material system was crystalline in nature, the cohesive behavior was extracted from the growth/deformation of an embedded pre-existing crack at the material interface. In material systems that contain amorphous thermoset polymer, the technique of embedded cracks/voids does not yield meaningful information pertaining to damage initiation.

Simulation of damage initiation at the nanoscale and the integration of relevant damage parameters to continuum scale damage models provide insight into damage precursors and the effects of applied loading. The linear elastic response of carbon nanotube (CNT)-enhanced epoxy polymers has been extensively studied; however, fundamental behavior at the molecular level causing inelastic response of the nanocomposite material has not been well understood. The mechanism of damage initiation is also significantly different in crystalline and amorphous materials. In a previous effort, the authors employed a hybrid force field MD simulation methodology to capture bond breakage in epoxy polymers [19]. This methodology was used to simulate damage caused by successive bond breakages using a combination of high strain rate and low temperature MD simulations and was compared to quasi-continuum (QC) simulations showing excellent correlation [20]. Thus, a computationally efficient numerical approximation to physics-based damage initiation phenomenon (QC-equivalent) in nanoparticle-embedded epoxy polymers was generated and implemented within a fully stochastic microscale damage framework [21,22]. The damage initiation model is integrated in this work for the characterization of the fiber/matrix interphase in the three-constituent system. 
This paper presents a methodology to characterize the interface between the fiber and the CNT-dispersed epoxy polymer in nanocomposites by integrating an atomistic model with a continuum model. As previously stated, MD simulations have tremendously contributed to the study of physical and chemical interactions in multiphase materials [23-25]. However, the high computational cost associated with the atomistic simulations limits their use beyond the nanoscale. Hence, the modeling of carbon/graphite fibers that have diameters in the order of microns is not feasible via MD simulations. In this paper, the relevant section of the fiber (at the interface) is modeled using irregularly stacked graphene layers with induced voids. The epoxy resin and hardener molecules penetrate these voids and form crosslink bonds in the presence of dispersed CNTs. Thus, the molecular model captures the physical entanglements formed by the polymer chains with graphene layers representing adhesion at the semi-crystalline fiber surface. It is noteworthy that existing literatures on nanoscale-driven cohesive law involving polymers only account for non-bonded van der Waals forces [10,26,27] and are not suitable for interfaces with functionalization or mechanical interlocking. Fiber pullout tests in this study are simulated by the relative sliding of the graphene layers with the CNT-dispersed polymer phase. The bonded and non-bonded interactions and the resulting geometric distortions at the three-constituent interphase during the pullout simulations are quantified to formulate a cohesive law for the fiber/matrix interface. The atomistically informed cohesive law can be integrated to a continuum micromechanics model to obtain the overall response at the microscale. This multiscale interface model is expected to be a valuable tool in identifying damage precursors at the interface in polymer matrix nanocomposites.

\section{Nanoscale Constituent Model}

The molecular structures of the epoxy resin and the hardener that constitute the polymer used in this study are DGEBF (Di-Glycidyl Ether of Bisphenol F) and DETA (Di-Ethylene TriAmine), respectively. The MD simulation package, Large-scale Atomic Molecular Massively Parallel Simulator (LAMMPS) [28], is used to perform the simulations. All-atom classical forcefields - OPLS (Optimized Potentials for Liquid Simulations) [29] and MMFF (Merck Molecular Force Field) [30], are implemented to obtain the atomic and molecular interactions causing elastic behavior in CNTs and the epoxy polymer, respectively. A stochastic crosslink formation approach, based on a cutoff value for covalent bond formation between active sites is adopted to simulate the epoxy curing process [25]. The probability distribution of crosslinking degree for 
the polymer system in the presence of CNTs has been presented in Ref [24]. In this paper, crosslinking degree of the epoxy resin and hardener molecules at the local interphase (where the polymer molecules are mechanically entangled with graphene layers) is estimated using molecular simulations.

Traditional MD simulations employed with an empirical force field are not capable of capturing bond dissociation between atoms leading to plasticity. Although MD simulations that employ an empirical force-field are useful to study processes in a system around its equilibrium state (e.g. curing), they are not appropriate for investigating covalent bond dissociation occurring away from the equilibrium state (e.g. during deformation tests). The external forces applied during a deformation test propagate through bonds in the matrix and in turn, the deformation induced by mechanical loading elongates the covalent bonds. It is therefore of interest to capture the effective local force in the deformed interphase to identify covalent bond dissociation, which characterizes the plasticity in the system at higher length scales. Hence, the bond order based potential parameter set from Singh et al. [31] is introduced to capture bond elongation and subsequent dissociation of covalent bonds in the interphase models during the virtual pullout tests. The novel QC-equivalent MD simulation approach using which covalent bond dissociation is captured and quantified at the interphase, is presented in detail in Ref $[19,20]$

\section{Fiber/Matrix/CNT Interphase}

This focus of the interphase model in this study is to investigate the three-constituent fiber/polymer/CNT interphase in carbon fiber-reinforced nanocomposites. The semi-crystalline structure of carbon fiber surface, which introduces roughness, is simulated by deliberately inducing defects in irregularly stacked crystalline, protruded graphene layers. The carbon fiber surface model is constructed by stacking several crystalline graphene layers with hydrogenated graphene layers with voids as shown in Figure 1. The graphene layers with induced voids simulate the irregularities and roughness on the carbon fiber surface. Note that typical carbon fiber surfaces contain residual nitrogen from polyacrylonitrile, oxidization residues, and compounds from surface treatment. However, this preliminary molecular investigation of the interphase assumes the fiber surface to be free from chemical residues other than terminal hydrogen atoms. Although the graphene layers appear to be perfectly stacked at the onset of the simulation, the energy minimization and equilibration processes in MD render them to be 
irregular, thereby capturing the surface waviness and roughness of the fiber. The model enables mechanical interlocking of the polymer molecules through the voids in the graphene layers. It is also capable of simulating the mechanical and chemical entanglement between the fiber and the matrix phases in the composite material.

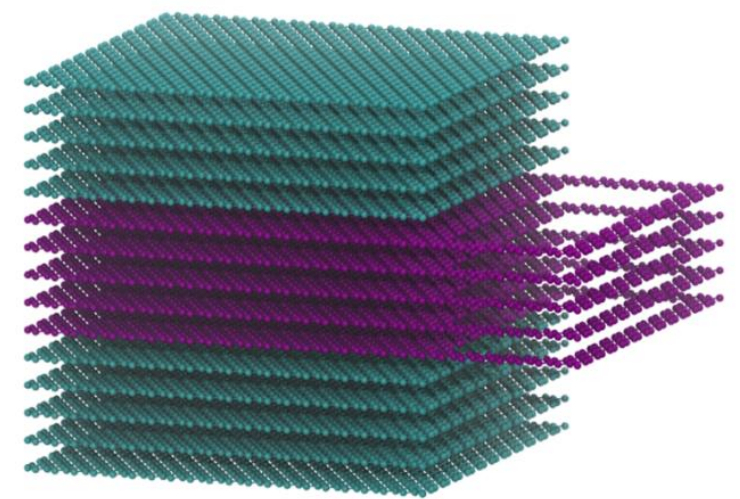

Figure 1. Schematic of carbon fiber surface (from [32])

Since the graphene layers with induced defects are not thermally stable, it is necessary to hydrogenate the defect sites before implementing them in the interphase model. The defects are induced by selectively removing $s p 2$ carbon atoms and activating them. The void induced graphene layer is equilibrated primarily at 300K, 1 atm for $50 \mathrm{ps}$ (controlled by a Berendsen thermostat and barostat) with the NPT (isobaric-isothermal) ensemble using a timestep of 1 fs. The sp 2 carbon atoms and the activated carbon atoms are differentiated by assigning different atom types. Hydrogen atoms are introduced in the simulation volume after equilibration, and a cutoff distance based bond formation methodology, similar to that used for the crosslinking of epoxy resin and hardener [25], is implemented to ensure the saturation of active sites with hydrogen. The cutoff distance used for bond formation is $9 \AA$, which is large enough to ensure that all the active sites are hydrogenated. Figure 2 illustrates the graphene layer with voids after the hydrogenation of active sites. 


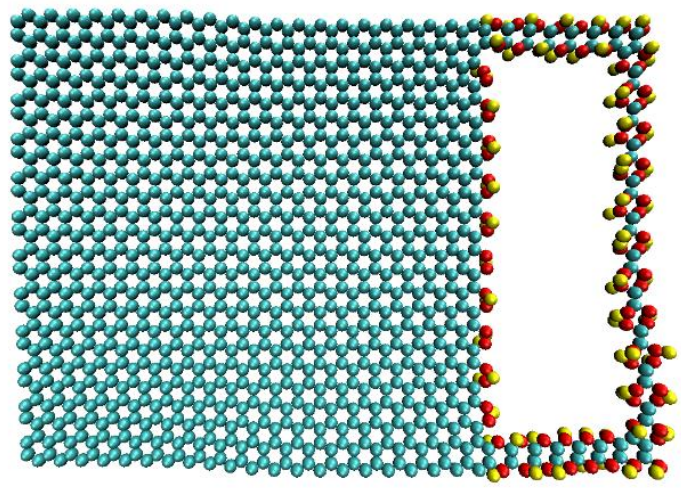

\section{Figure 2. Hydrogenated graphene layer}

The pristine graphene layers and the hydrogenated graphene layers are stacked irregularly, and the epoxy resin and hardener molecules are dispersed in the simulation volume with the CNT molecules protruding out in the $\mathrm{x}$-direction. This arrangement of CNT represents a configuration with radially grown CNT on the fiber surface, similar to that of a fuzzy fiber architecture [33]. The numerical crosslinking of the resin and hardener allows the polymer network to penetrate the voids in the graphene layers capturing entanglement between the carbon fiber surface and the polymer matrix. This entanglement of polymer chains with the graphene layers acts as a mechanical reinforcement in addition to the non-bonded interactions simulated by the Lennard-Jones potential. It is important to note that the MD simulations of the interphase models are designed to prevent the polymer chains from penetrating the graphene layers. The initial dimensions of the interphase model are $130 \times 100 \times 50 \AA^{3}$, containing 58,000 atoms in the simulation volume. Periodic boundary conditions are applied along the $\mathrm{y}$ - and $\mathrm{z}$-directions; however, the simulation is considered to be non-periodic along the $\mathrm{x}$ direction. Energy minimization is performed using the conjugate gradient method followed by NPT ensemble equilibration is performed at $300 \mathrm{~K}$ and 1 atm for $10 \mathrm{~ns}$ (1fs timestep) controlled by a Berendsen thermostat/barostat. The cutoff distance based method is applied to the equilibrated model to generate crosslink bonds between the carbon atoms in the resin and the nitrogen atoms in the hardener ( $\mathrm{C}-\mathrm{N}$ bond). The defined cut-off distance is $4 \AA$, which is approximately the sum of the van der Waals radii of $\mathrm{C}$ and N. Figure 3 illustrates the cured interphase model and the corresponding loading conditions for the different modes of loading used to characterize the fiber/matrix interphase. Figure 3(a) represents a virtual pullout test where the left ends of all the graphene layers are constrained along the x-direction, allowing movement only in the $\mathrm{y}$ - and $\mathrm{z}$ directions. A displacement boundary condition is applied to the polymer matrix along the $\mathrm{x}$-axis 
with the CNT molecule following the displacement of the polymer chains $(0.0001 \AA / f s)$. Figure 3(b) illustrates the fiber shear mode where the polymer phase is displaced along the y-direction $(0.0001 \AA / f s)$. During the shear displacement, the stacked graphene layers are allowed to follow the polymer phase, thus simulating fiber rotation under shear. In Figure 3(c) the boundary conditions for a simple fiber pullout test are depicted in which the fiber phase moves relative to a stationary polymer phase along the z-direction.

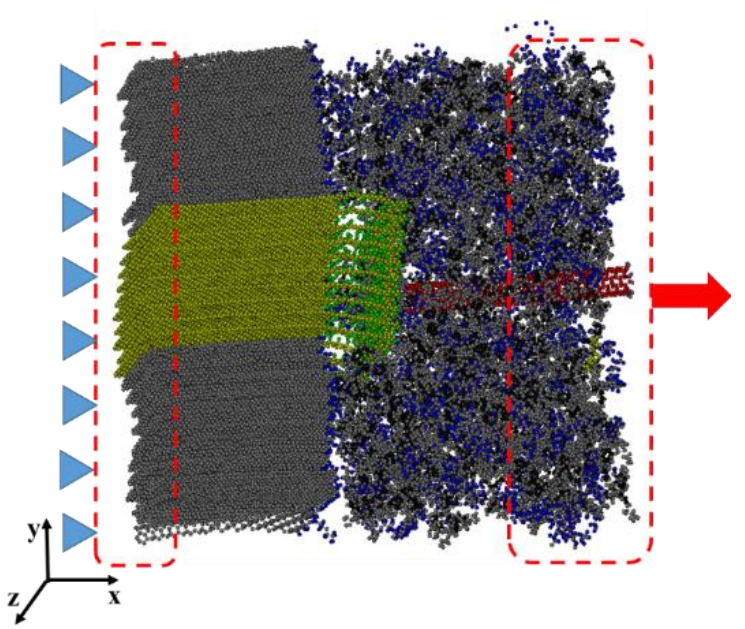

(a)

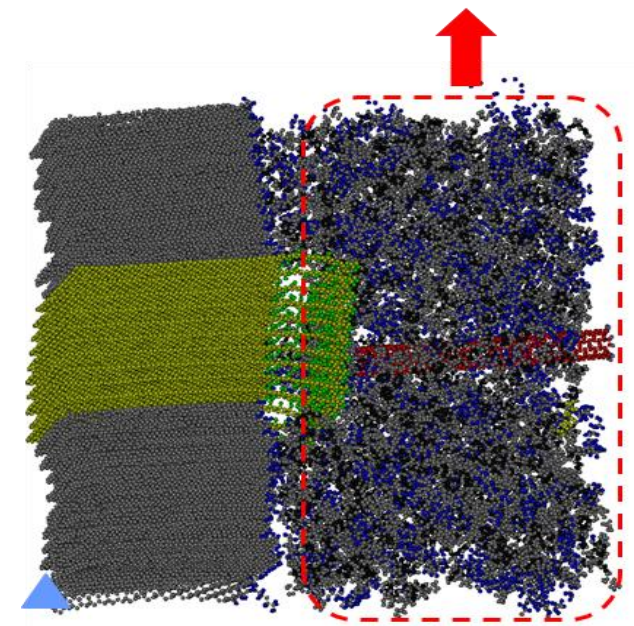

(b)

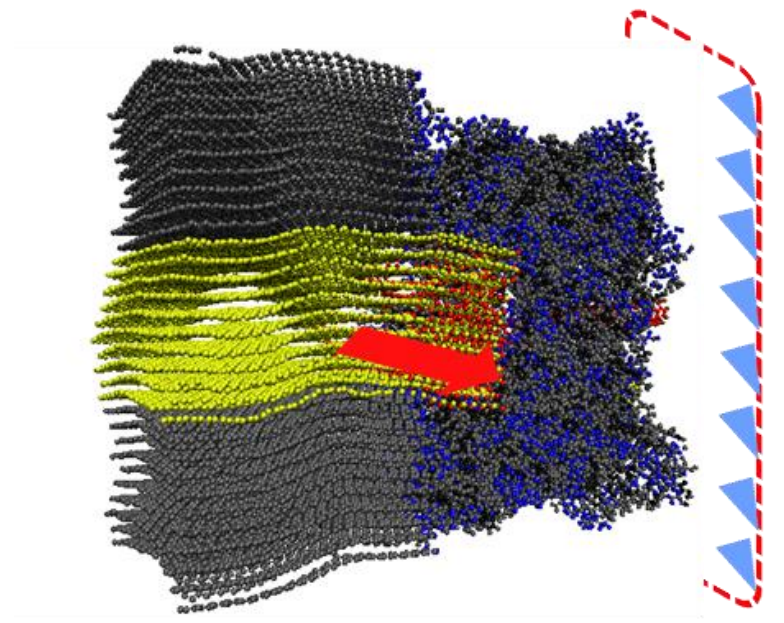

(c)

Figure 3. Loading and boundary conditions on the fiber/matrix interphase model (a) mode-

\section{I loading; (b) mode-II loading; (c) mode-III loading}

The local curing (numerical crosslinking) of the epoxy polymer in the vicinity of the interphase is investigated first. A stochastic distribution of the crosslinking degree for the neat epoxy polymer, used in this study, was generated in the authors' recent work [24]. The most likely crosslinking degree of neat epoxy unit cells was found to be $56.02 \%$ through MD 
simulations. Following a similar procedure, the mean crosslinking degree of the polymer in local regions near the interphase is determined to be $30.15 \%$ from multiple MD simulations with different initial configuration of molecules. This reduction in the crosslinking degree shows that the presence of graphene layers and CNT at the interphase impedes the formation of crosslink bonds in the polymer molecules, thus, making the polymer network weaker. The weaker interactions at the molecular level can scale up to the fiber/matrix interface region rendering this region weaker and prone to damage initiation.

The stress-strain curve from a virtual pullout test is shown in Figure 4. The transverse pullout simulations are performed on multiple unit cells with different initial configuration of molecules. The average modulus of the interphase is determined to be $9.69 \mathrm{GPa}$ with a standard deviation of $0.84 \mathrm{GPa}$. The elastic response of the interphase is limited to a strain of $\sim 3.9 \%$. The elastic region corresponds to the polymer chains being stretched under the displacement load. The sudden stress drop following the yield point indicates the quick dissociation of weak bonds in polymer network. Subsequently, a hardening phase is observed as a result of the mechanically entangled polymer chains stretching further under the load. This mechanism leads to effective load transfer between the polymer chains, the CNT and the carbon fiber surface. The strain hardening extends to $\sim 16 \%$ strain, following which, the polymer chains break in quick succession leading to the complete failure of the interphase. The intermediate fluctuations in the stress-strain curve could be attributed to local failure arising from chain breakages in regions of poor epoxy crosslinking density.

The bonded and non-bonded interactions at the interphase are also monitored during the pullout simulations. Figures 5(a) and (b) show the variation of pair/van der Waals energy and bond energy during the simulations, respectively. The consistent rise in non-bonded pair energy could be attributed to the breakage of covalent bonds, converting bonded interaction to a nonbonded interaction if the atoms previously involved in the covalent bond are within the van der Waals cutoff distance. The final drop in pair energy is due to the total failure of the interphase due to massive bond dissociation and atoms escaping the field of van der Waals radius. 


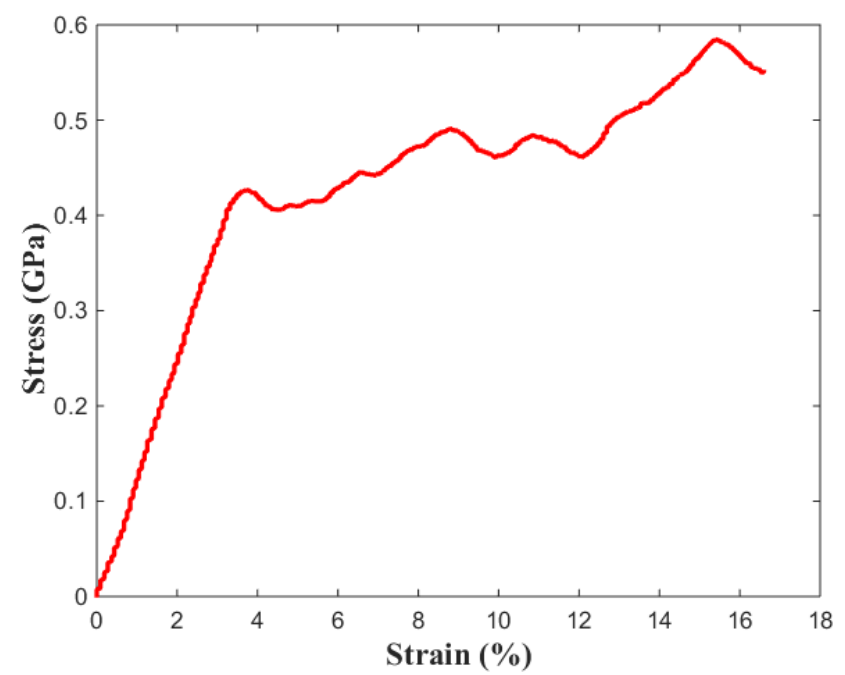

Figure 4. Stress-strain response during interphase pullout test

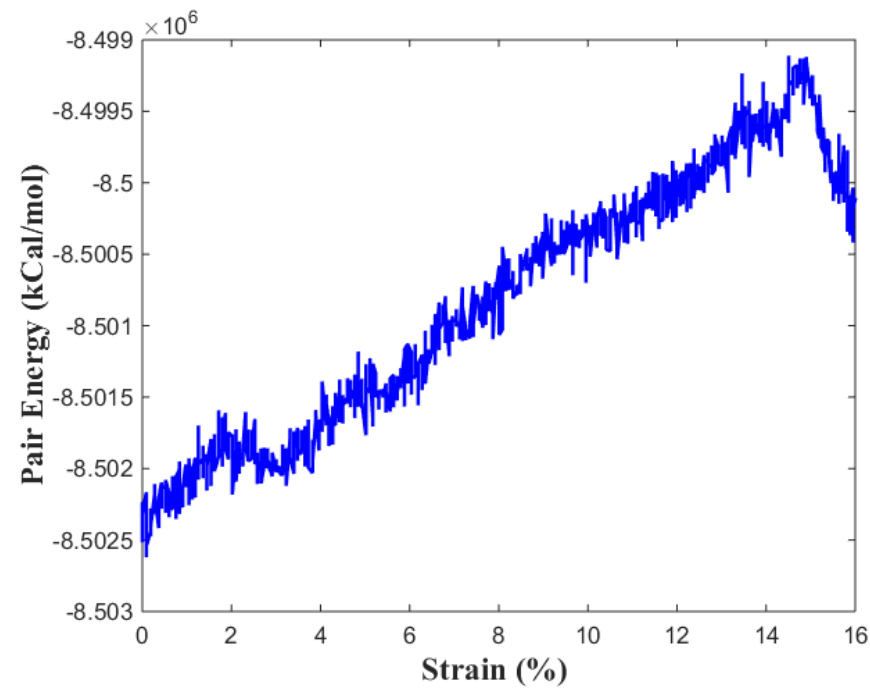

Figure 5(a). Pair energy variation during pullout simulation 


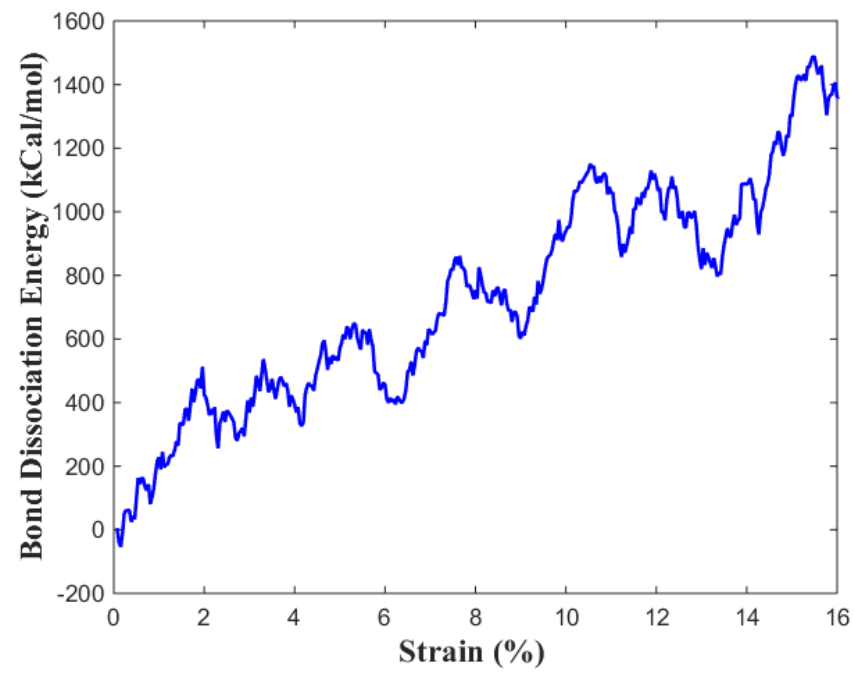

Figure 5(b). Bond dissociation energy during pullout simulation

The extension, relaxation, and dissociation of bond clusters are captured by the variation in bond dissociation energy (BDE), which represents the variation of bond energy with reference to the ground state. The bond energy corresponding to the bound, ground state of the interphase after equilibration is set to be zero. The increase in BDE indicates bonds breakage under the applied load; the fluctuations are due to relaxation of local bond clusters subsequent of bond breakage in chains.

\section{Atomistically Informed Cohesive Law for Interphases}

The data from molecular interphase simulations can be integrated into a continuum model by defining a cohesive traction-separation law for the fiber/matrix interface. The cohesive zone model implemented in this study is a modified formulation of the equations derived by Tvergaard [34], which enables the linking between cohesive zone parameters to equivalent plastic strain and void growth processes. The response of the fiber/matrix interface that needs to be captured by the cohesive law is obtained from the MD simulations of the interphase debonding, fiber shear, and fiber pullout modes. In continuum models involving CZM, the cohesive stresses are dominant until the interface fails. Subsequent to interface failure, the mechanics of the interface is dictated by the frictional forces. However, in MD, the interactional potentials between the phases cause variations in potential energy during pullout/deformation that manifest as cohesion and friction at the continuum level. Therefore, the cohesive forces in 
this study are calculated as negative gradients of potential energy due to non-bonded van der Waals interactions and bonded distortions.

The cohesive behavior of the fiber/CNT-dispersed matrix phases is dependent on the debond mode. Mode-I debonding occurs when the matrix separates from the fiber in tension (as shown in Figure 3a), equivalent to mode-I crack-opening in fracture mechanics. Mode-III debonding results from a fiber pullout along the length of the fiber due to relative shear loading. Both modes of failure are simulated using the fiber/matrix interphase molecular model presented in the previous section. Figures $6-8$ illustrate the force-displacement relationship in the molecular system under mode-I, mode-II, and mode-III loading conditions, respectively. Note that the mode-I, mode-II and mode-III deformation simulations are performed on five random initial molecular configurations. The results in Figures $6-8$ are the averaged response from the five configurations.

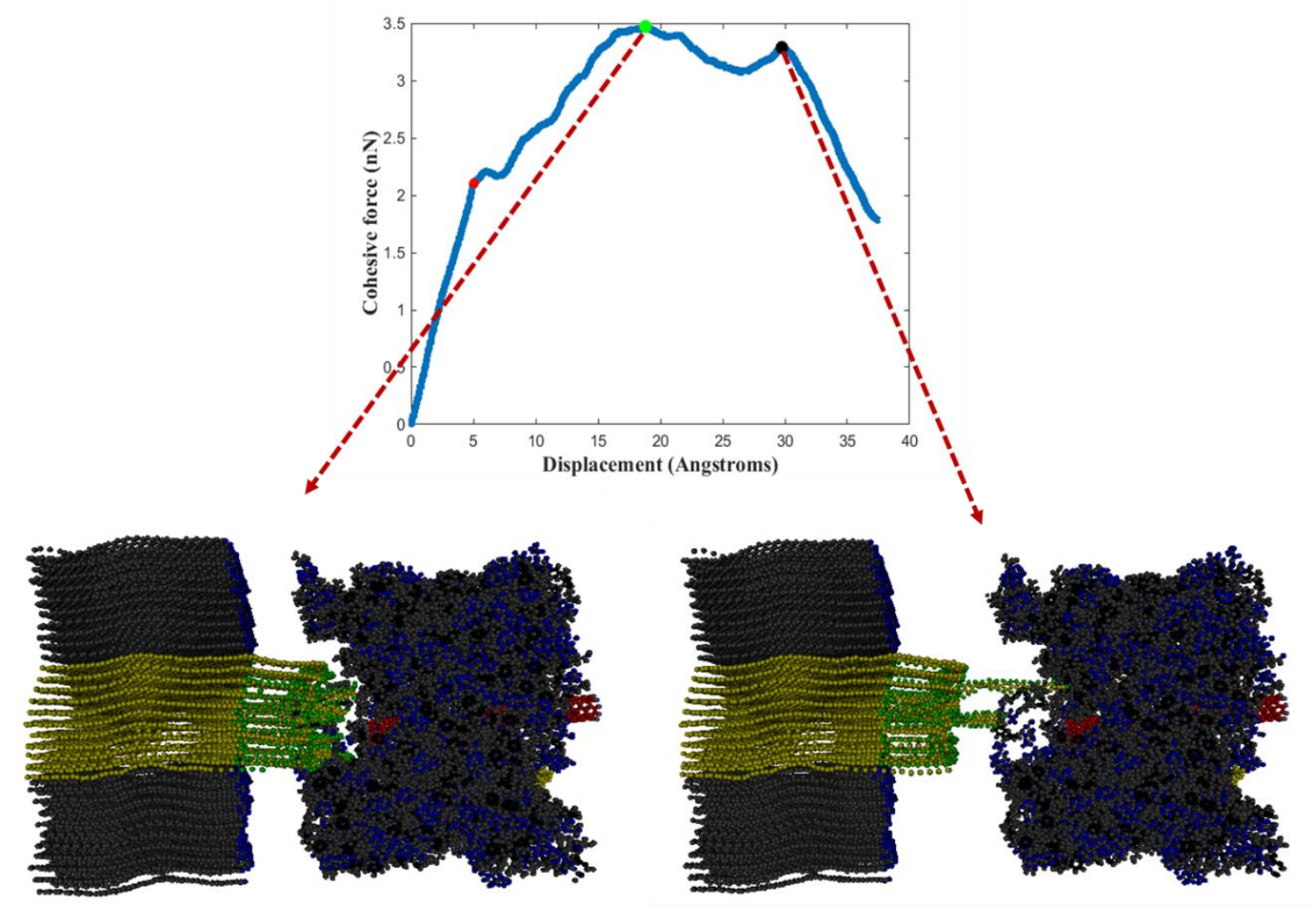

Figure 6. Force-displacement response under mode-I loading causing matrix debond 


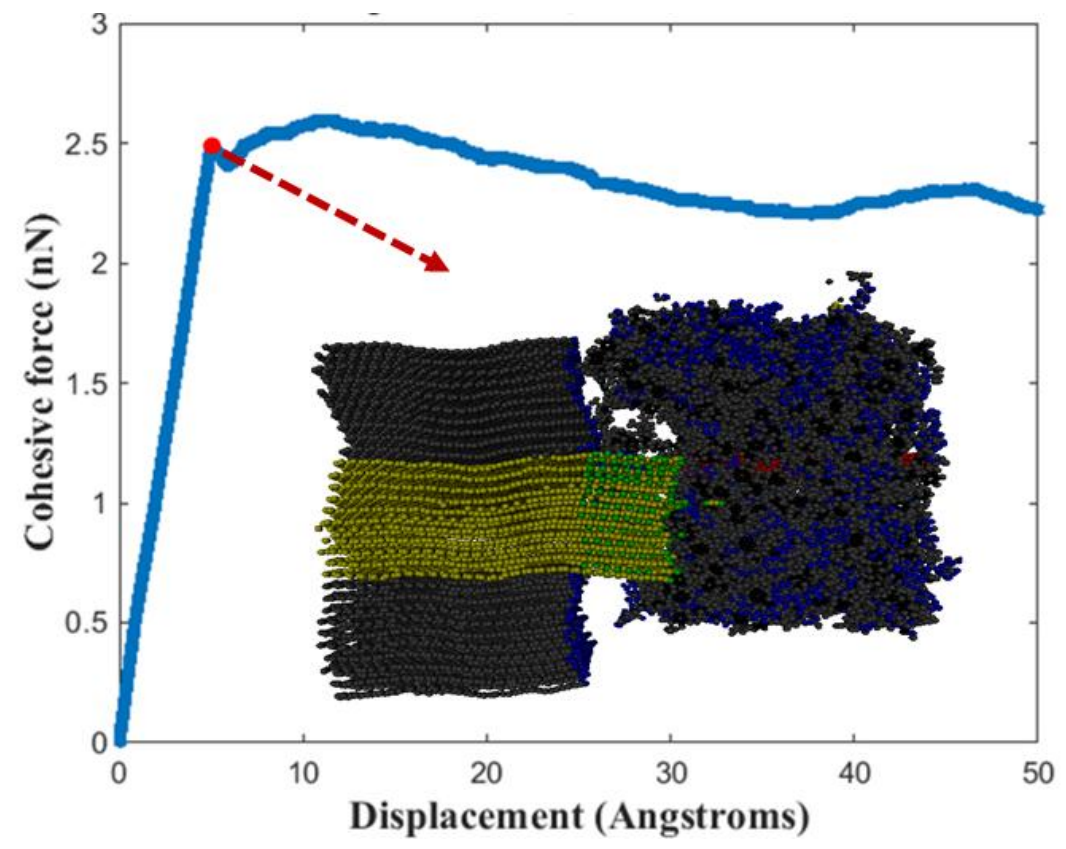

Figure 7. Force-displacement response under mode-II loading causing fiber rotation

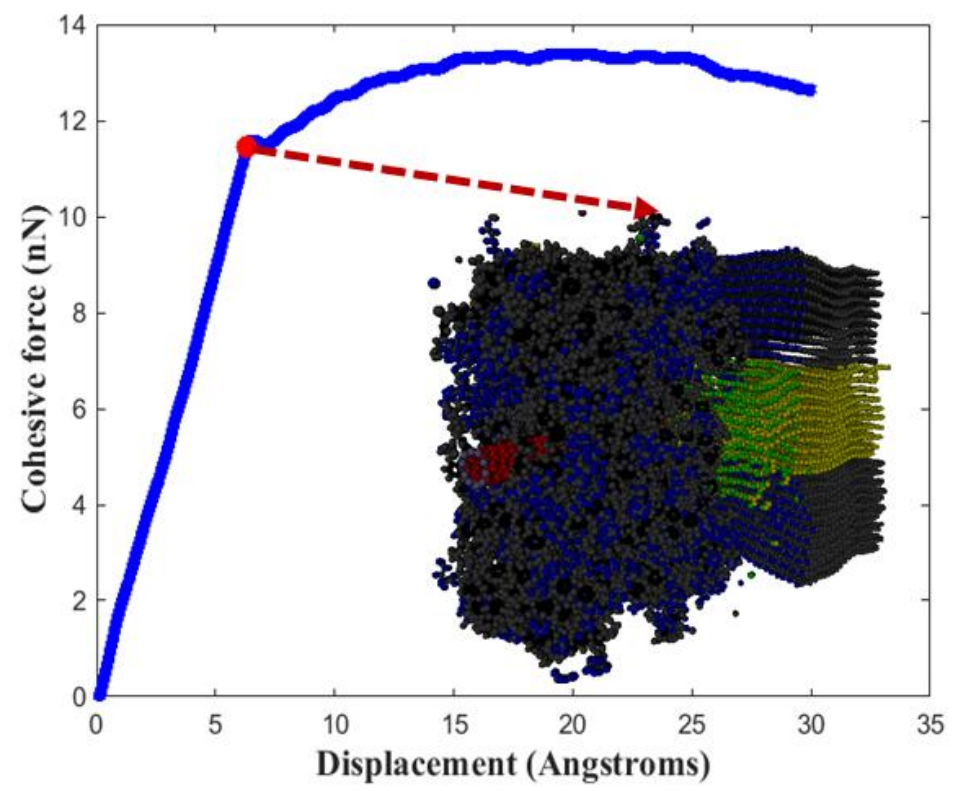

Figure 8. Force-displacement response under mode-III loading causing fiber pullout

As shown in Figure 6, the cohesive behavior of the fiber/CNT-dispersed matrix interphase under mode-I loading exhibits a bimodal relationship between the interfacial tractions and the corresponding opening displacements. Under mode-II and mode-III loading, Figures 7 and 8 respectively, the cohesive behavior appears to be approximately trapezoidal in nature. Note that the mode-II and mode-III loading were performed along a periodic boundary ( $\mathrm{y}$ - and $\mathrm{z}$-direction, 
respectively) of the molecular unit cell. Hence, the final drop in load corresponding to total failure of the interphase is not captured.

Figure 6 shows the nature of interphase response during matrix debond from the fiber, transverse to the fiber direction. The first kink (red dot) in the plot corresponds to the yield point when the weak bonds in the interphase break. However, this breakage of weak bonds does not occur as a cluster in the unit cell. Therefore, the yield point is followed by a strain-hardening region where the polymer chains in the mechanically entangled interphase elongate under the applied load. The first drop (green dot) in the cohesive force occurs at a displacement of $\sim 17 \AA$. This corresponds to the breaking of physically entangled polymer chains at the graphene/polymer interphase. Subsequently, the polymer chains slide along the $\mathrm{x}$-direction (see Figure 3) and the loads are transferred to the remaining bulk of the polymer chains, leading to the second hardening region. The final drop (black dot) in load indicates the failure of the polymer phase itself indicating no more possibility of load transfer to the fiber (graphene layers). The degree of surface roughness in the fiber can be represented by the size of the void regions in the current molecular interphase model. The results indicate that an increase in surface roughness (larger voids in graphene layers) would lead to a higher spacing between the two peaks in the cohesive behavior. A higher degree of entanglement would result in a higher peak value of cohesive forces.

Figure 7 illustrates the response of the fiber-matrix interphase under mode-II loading. This loading represents relative shear along the y-direction, capturing the rotation of the fiber leading to failure. The critical cohesive force (red dot) corresponds to the bond breakage in the entangled region causing local interphase failure. Post the local failure event, the cohesive force gradually decreases allowing the relative rotation of the fiber with ease. The response along the $\mathrm{z}$-direction, representing mode-III shear loading leading to a fiber pullout is illustrated in Figure 8. The pullout force remains fairly constant once the yield point (red dot) is crossed. This is because the mechanical interlocking of the polymer chains with the graphene layers is broken at the yield point. Following this, the polymer chains slide smoothly in shear relative to the irregular graphene layers. From a continuum perspective, this is similar to overcoming the static friction (or adhesion) between the two phases. Once the static friction is overcome, the two phases slide with respect to each other under dynamic friction. It is noteworthy that the peak cohesive forces in mode-III are significantly higher than in the mode-I and mode-II responses. This is due to the 
fact that the fiber (graphene layers) is the major load carrying phase under mode-III loading whereas the matrix (polymer chains with CNT) is the major load carrying constituent phase under mode-I. Under mode-II loading, although the fiber is a major load carrying constituent, the load is transverse to the fiber orientation, and it is well known that fibers are weak in the transverse direction.

The cohesive formulations are developed to represent the traction-separation response under the different modes of loading. With $\left[u_{i}\right]$ as the opening/sliding displacements along the loading direction, $T_{i}$, the corresponding tractions, $T_{i_{\max }}$ the maximum traction, and $\delta i$ the critical displacement, the interface tractions are represented by:

$$
T_{i}=\frac{\left[u_{i}\right]}{\delta i} T_{i_{\max }} F(\psi, \varnothing, \theta)
$$

If the interphase at the continuum scale is represented by a very thin layer of interphase elements of thickness $h$, and further assuming uniform strains along the thickness, the strains are expressed as:

$$
\left\{\varepsilon_{11} \varepsilon_{12} \varepsilon_{13}\right\}=\frac{1}{h}\left\{u_{1} u_{2} u_{3}\right\}
$$

The critical strains are also defined as:

$$
\left\{\varepsilon_{11}^{c} \varepsilon_{12}^{c} \varepsilon_{13}^{c}\right\}=\frac{1}{h}\left\{\delta_{1} \delta_{2} \delta_{3}\right\}
$$

Equation 1 is then modified to obtain the constitutive law:

$$
\sigma_{i j}=\frac{\varepsilon_{i j}}{\varepsilon_{i j}^{c}} \sigma_{\max } F(\psi, \emptyset, \theta)
$$

The function $F(\psi, \emptyset, \theta)$ describing the overall cohesive behavior with $\alpha$ as the mode mixity parameter is obtained by the superposition of cohesive responses under each loading mode. In Equation 5, functions $\psi\left(u_{1}\right), \varnothing\left(u_{2}\right), \theta\left(u_{3}\right)$ describes the mode-I, mode-II and mode-III cohesive behavior, respectively.

$$
F(\psi, \emptyset, \theta)=\alpha_{1} \psi\left(u_{1}\right)+\alpha_{2} \emptyset\left(u_{2}\right)+\left(1-\alpha_{1}-\alpha_{2}\right) \theta\left(u_{3}\right)
$$

As an initial attempt, due to the nature of the mode-I response, $\psi\left(u_{1}\right)$ is represented using a bimodal approximation. In order to employ a bimodal Gaussian function, the data is transformed to a univariate space $\left(u_{1}, \psi\left(u_{1}\right)\right)$ and the corresponding histogram is obtained. The general equation of a bimodal Gaussian distribution is shown in Equation 6.

$$
\psi\left(u_{1}\right)=p\left(\frac{1}{\sqrt{\left(2 \pi S_{1}^{2}\right)}} \exp \left[-\frac{\left(u_{1}-\mu_{1}\right)^{2}}{2 S_{1}^{2}}\right]\right)+(1-p)\left(\frac{1}{\sqrt{\left(2 \pi S_{2}^{2}\right)}} \exp \left[-\frac{\left(u_{1}-\mu_{2}\right)^{2}}{2 S_{2}^{2}}\right]\right)
$$


The values of $\mu_{1}$ and $\mu_{2}$ correspond to the means of the two normal distributions; physically, this corresponds to the points of maximum traction causing local interphase failure initiation (green dot) and total loss of load transfer capacity (black dot) in Figure 6. The $S_{1}$ and $S_{2}$ correspond to the root mean square (RMS) width of the two Gaussian functions. From a physicsbased perspective, the RMS width deviation relates to the degree of entanglement of the molecular interphase; the higher the mechanical interlocking between the polymer chains and the graphene layers, the higher will be the RMS width of each distribution. Figure 9 shows the approximated bimodal Gaussian function.

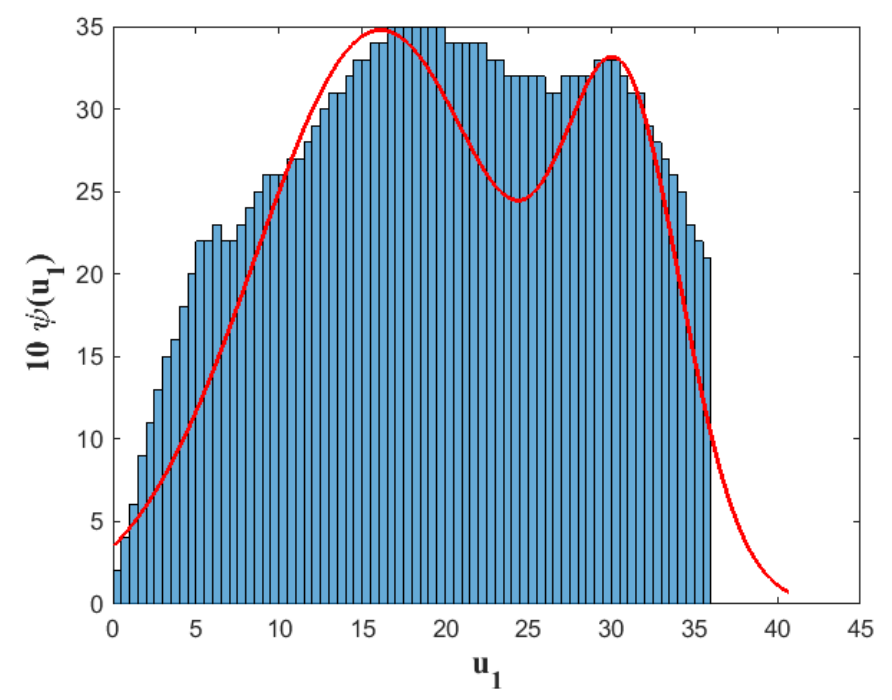

Figure 9. Bimodal approximation to mode-I cohesive behavior

The approximation of the bimodal Gaussian function applied to the mode-I data yielded the displacement values corresponding to $\mu_{1}$ and $\mu_{2}$ of $16.007 \AA$ and $30.67 \AA$, respectively. The $S_{1}$ and $S_{2}$ values were calculated to be 7.47 and 3.62, respectively, and the $p$ value to be 0.722 . Although the bimodal function is able to capture the maximum tractions and their corresponding critical displacements accurately, the tractions in the region between the local interphase failure and complete loss of load transfer is underestimated. The initiation of failure is captures accurately using the bimodal Gaussian function.

A bivariate polynomial function is also explored to approximate the mode-I tractionseparation behavior. In order to retain the physics captured by the MD simulations, the quadratic function is imposed in meaningful piecewise regions (see Figure 10). 


$$
\psi\left(u_{1}\right)=\left\{\begin{array}{c}
a_{1} u_{1}^{2}+b_{1} u_{1}+c_{1} \text { if } u_{1} \leq \delta_{1_{c}} \\
a_{2} u_{1}^{2}+b_{2} u_{1}+c_{2} \text { if } \delta_{1_{c}}<u_{1} \leq \delta_{1 f} \\
b_{3} u_{1}+c_{3} \text { if } u_{1}>\delta_{1_{f}}
\end{array}\right.
$$

The parameters of the function are obtained through a constrained minimization algorithm forcing the curves to pass though the break points of the piecewise sections, and imposing continuity constrains at the break points (numerical continuity through a tolerance condition).

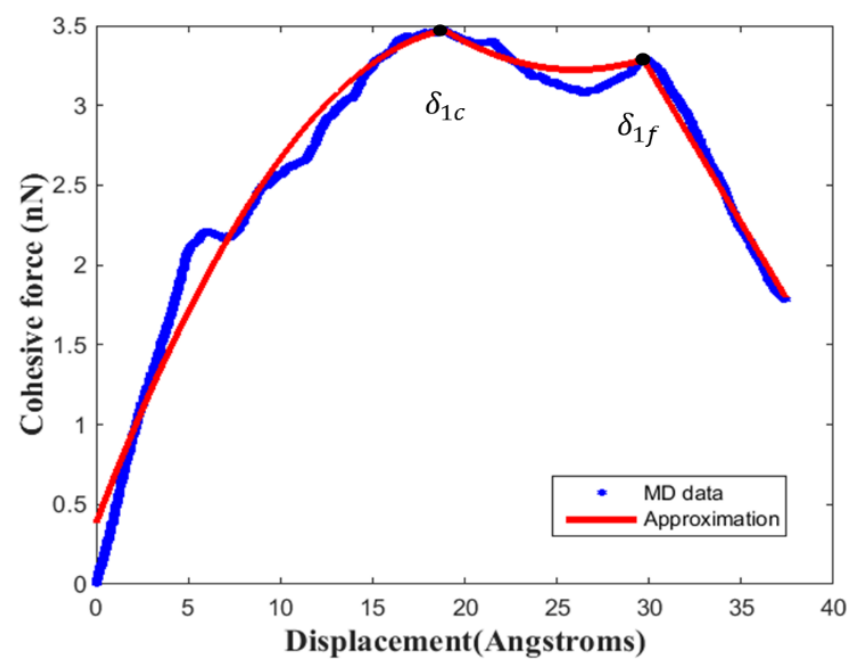

Figure 10. Piecewise polynomial approximation to mode-I cohesive behavior

Trapezoidal distributions are used to approximate the functions $\emptyset\left(u_{2}\right)$ and $\theta\left(u_{3}\right)$ due to the nature of shear loading and the results from Figures 7 and 8 . The mode-II/mode-III response is also represented using piecewise approximation by identifying meaningful cutoff regions. The response is a trapezoidal traction-separation law with the critical displacement $\left(\delta_{2 c}, \delta_{3 c}\right)$ representing the point where static friction is overcome, and parameters $\delta_{2 f}, \delta_{3 f}$ indicating loss of load transfer capability between the fiber and the matrix (see Figures $11 \& 12$ ). As mentioned earlier, the mode-II and mode-III loading were performed under periodic boundary constraints; hence, the final drop in load corresponding to total failure is not captured by these formulations.

$$
\varnothing\left(u_{2}\right)=\left\{\begin{array}{c}
p_{1} u_{2}+q_{1} \text { if } u_{2} \leq \delta_{2 c} \\
p_{2} u_{2}+q_{2} \text { if } \delta_{2}<u_{2} \leq \delta_{2 f} \\
p_{3} u_{2}+q_{3} \text { if } u_{2}>\delta_{2 f}
\end{array}\right.
$$




$$
\theta\left(u_{3}\right)=\left\{\begin{array}{c}
m_{1} u_{3}+n_{1} \text { if } u_{3} \leq \delta_{3 c} \\
l_{2} u_{3}^{2}+m_{2} u_{3}+n_{2} \text { if } \delta_{3}<u_{3} \leq \delta_{3 f} \\
m_{3} u_{3}+n_{3} \text { if } u_{3}>\delta_{3 f}
\end{array}\right.
$$

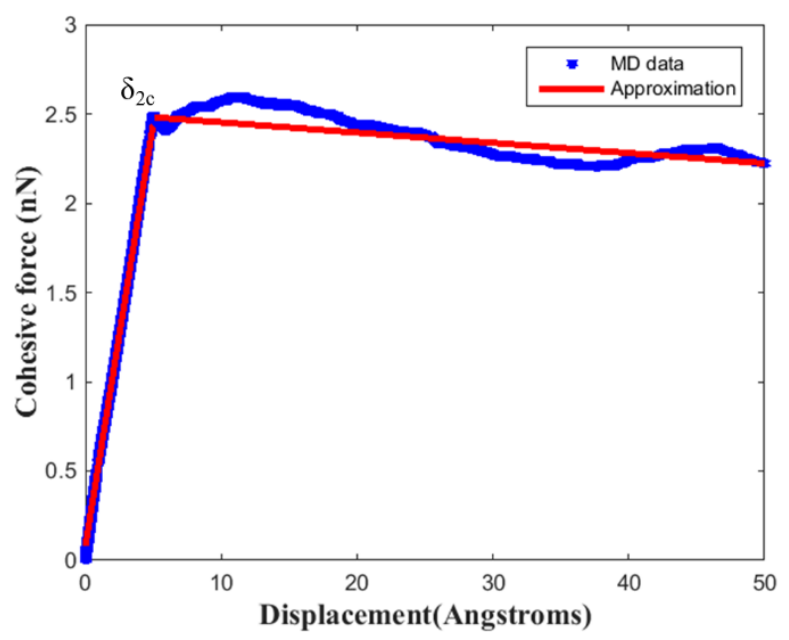

Figure 11. Piecewise polynomial approximation to mode-II cohesive behavior

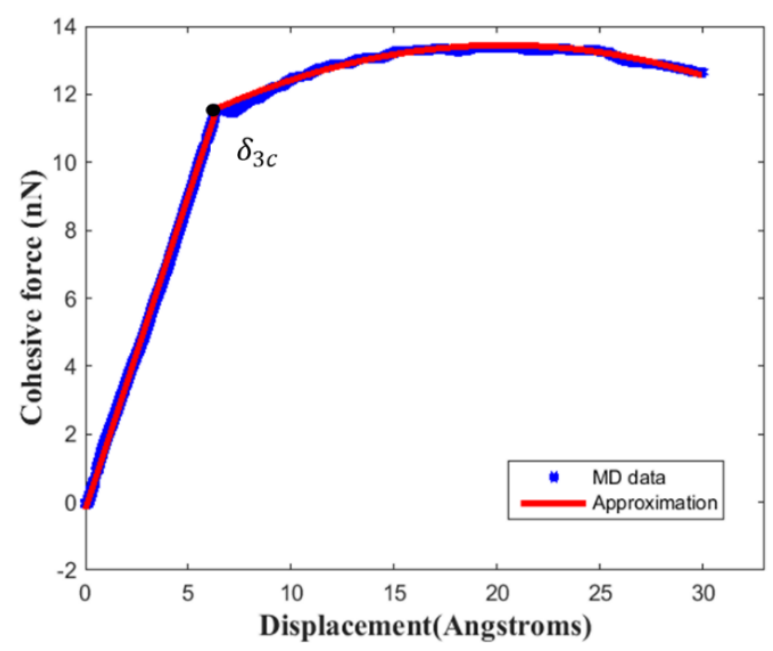

Figure 12. Piecewise polynomial approximation to mode-III cohesive behavior

The simulation results provide a comprehensive understanding of the interphase mechanics which is driven by nanophysics. Experimental studies on matrix debonding performed at the macroscale involve a pre-existing crack to characterize the interface cohesive mechanics. Finite element (FE) models with cohesive elements [35] and XFEM studies [36] often utilize a bilinear cohesive law to fit the experimental curve. The bilinear cohesive law fails to capture damage initiation at the fiber/matrix interface and only accounts for damage propagation. Figure 6 , on the other hand, captures the traction causing damage initiation at the local interphase and its 
propagation leading to interphase failure through matrix debond. Micromechanics [10,27] and MD based cohesive laws in literature [18,37] for debond in finite thickness interphases exhibit quadratic or cubic behavior with a single peak. The bimodal behavior is not captured due to the exclusion of bonded distortions in these studies. Especially in the matrix debond mode, the inclusion of bonded distortions is critical because the bonded effects dominate the failure mechanism; as the deformation of the interphase progresses, the non-bonded van der Waals interactions diminish with distance.

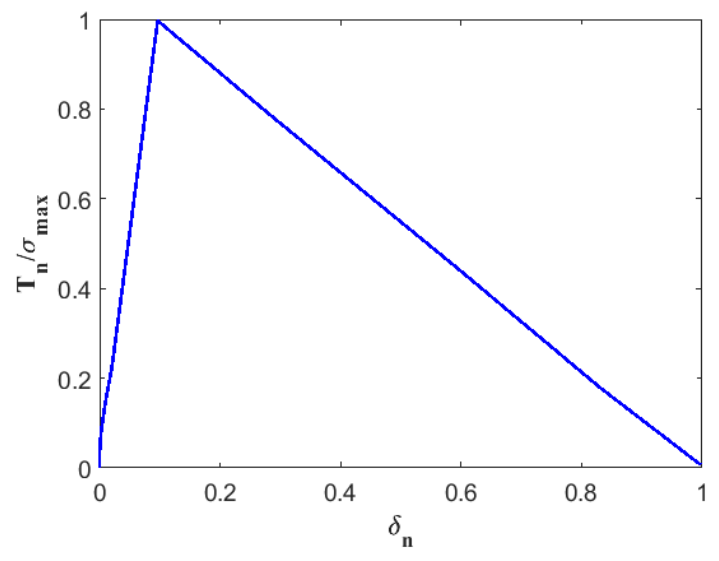

(a)

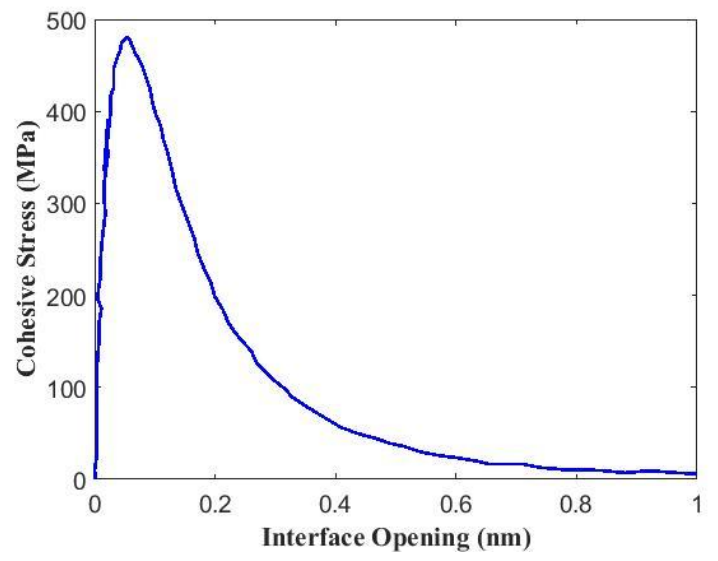

(b)

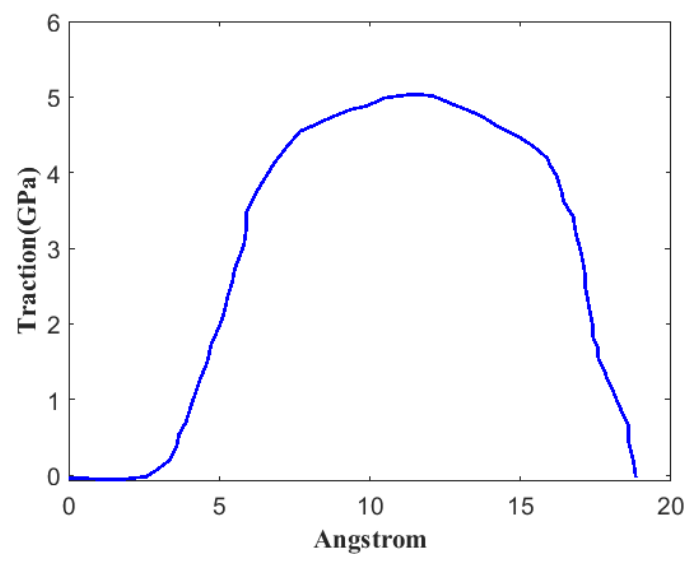

(c)

Figure 13. (a) Cohesive law for normal tractions in fiber debonding with friction (data from [35]); (b) Cohesive law for CNT-polymer interface based on van der Waals interactions (data from [27]); (c) Traction separation from atomic simulations for CNTepoxy interface (data from [18]) 
The fiber pullout mode, however, is dominated by van der Waals forces subsequent to the dissociation of entangled networks at the interphase because the distance between the two phases remains fairly constant. Therefore, the MD studies with only non-bonded interactions [18] are able to capture the trapezoidal behavior exhibited in Figure 8. This also shows good correlation with experimental results from a single fiber pullout test [38].

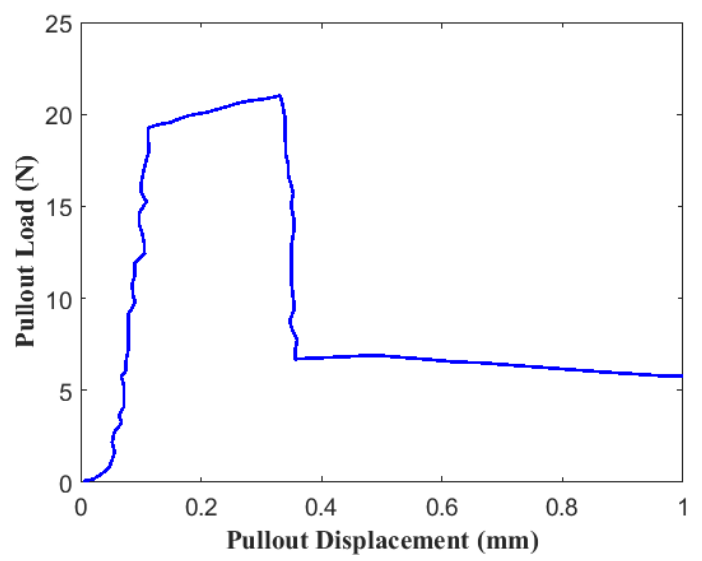

Figure 14. Trapezoidal traction-separation behavior obtained from experiments during fiber pullout (data from [38])

The explicit multimodal traction-separation curves form the basis of analytical equations, which can be used to calculate the failure load for the fiber/matrix interphase. This formulation also shows potential to be used in conjunction with numerical methods such as with FE analysis. Interphase elements that are defined by the constitutive behavior represented in Equation 4 can be developed using user material subroutines and commercial finite element solvers. A significant advantage of this approach is that the cohesive law can be incorporated within any multiscale framework for computational nanocomposite analysis, including the one developed by the authors $[21,22]$. Such a framework can be used to study damage initiation and propagation at the interface, fiber debonding, fiber pullout, matrix cracks in the polymer and the interaction of matrix damage with the interphase damage which lead to rapid structural failure.

\section{Conclusion}

A computational methodology was developed to characterize the molecular interphases in carbon fiber reinforced CNT/epoxy nanocomposites. Hybrid force field MD simulations were implemented to study near-equilibrium and bond breakage effects. A novel fiber/matrix molecular interphase was generated using stacked graphene layers enabling mechanical 
entanglement between the fiber surface and the polymer chains. The transverse modulus and nonlinear response of the fiber/matrix interphase were determined by virtual pullout MD simulations under three different modes of loading. Results from the molecular interphase models indicate that the penetration of polymer chains through the voids in the irregular fiber surface leads to stronger bonded and non-bonded interactions. The variation of pair energy and BDE were quantified during the transverse pullout simulation.

Cohesive behavior at the interphase was characterized in order to formulate a tractionseparation curve; the equations to non-parameterize the traction separation curves are presented. The molecular modeling of the interphase provided insight into the entanglement phenomena and variations in the cohesive behavior that have not been captured by micromechanical approximations or studies in literature that have disregarded bond distortion effects. The nonparameterized traction-separation behavior can be included in continuum FE-based models to understand damage initiation and propagation at the fiber/matrix interface in carbon fiber nanocomposites.

\section{Acknowledgment}

This research is supported by the Office of Naval Research (ONR), Grant number: N0001414-1-0068. The program manager is Mr. William Nickerson. The authors also acknowledge Dr. Anisur Rahman, a technical liaison for this research and the DoD ERDC Supercomputing Resource Center.

\section{References}

[1] Datta S, Fard MY, Chattopadhyay A. High-Speed Surfactant-Free Fabrication of Large Carbon Nanotube Membranes for Multifunctional Composites. J Aerospace Eng 2015;29:04015060.

[2] Hasan Z, Chattopadhyay A, Liu Y. An investigation into the performance of composite hat stringers incorporating nanocomposites using a multiscale framework. J Reinf Plast Compos 2014:0731684414529832.

[3] Datta S, FARD M, JOHNSTON J, Quigley E, Chattopadhyay A. In-situ Strain and Damage Sensing in Glass Fiber Laminates Using Embedded CNT. Structural Health Monitoring 2015 2015.

[4] Hasan Z, Chattopadhyay A. Thermo-Mechanical Analysis of Structural Elements Incorporating Nanocomposites. 2013:V009T10A084-. 
[5] Truong HT, Lagoudas DC, Ochoa OO, Lafdi K. Fracture toughness of fiber metal laminates: Carbon nanotube modified Ti-polymer-matrix composite interface. J Composite Mater 2013:0021998313501923.

[6] Rafiee MA, Rafiee J, Srivastava I, Wang Z, Song H, Yu Z et al. Fracture and fatigue in graphene nanocomposites. small 2010;6:179-83.

[7] Rafiee MA, Rafiee J, Wang Z, Song H, Yu Z, Koratkar N. Enhanced mechanical properties of nanocomposites at low graphene content. ACS nano 2009;3:3884-90.

[8] Gou J, Minaie B, Wang B, Liang Z, Zhang C. Computational and experimental study of interfacial bonding of single-walled nanotube reinforced composites. Computational Materials Science 2004;31:225-36.

[9] Wagner HD. Nanotube-polymer adhesion: a mechanics approach. Chemical Physics Letters 2002;361:57-61.

[10] Jiang L, Huang Y, Jiang H, Ravichandran G, Gao H, Hwang K et al. A cohesive law for carbon nanotube/polymer interfaces based on the van der Waals force. J Mech Phys Solids 2006;54:2436-52.

[11] Odegard GM, Frankland SV, Gates TS. Effect of nanotube functionalization on the elastic properties of polyethylene nanotube composites. AIAA J 2005;43:1828-35.

[12] Odegard G, Clancy T, Gates T. Modeling of the mechanical properties of nanoparticle/polymer composites. Polymer 2005;46:553-62.

[13] Katnam K, Sargent J, Crocombe A, Khoramishad H, Ashcroft I. Characterisation of moisture-dependent cohesive zone properties for adhesively bonded joints. Eng Fract Mech 2010;77:3105-19.

[14] Turon A, Davila CG, Camanho PP, Costa J. An engineering solution for mesh size effects in the simulation of delamination using cohesive zone models. Eng Fract Mech 2007;74:1665-82.

[15] Song SH, Paulino GH, Buttlar WG. A bilinear cohesive zone model tailored for fracture of asphalt concrete considering viscoelastic bulk material. Eng Fract Mech 2006;73:2829-48.

[16] Roe K, Siegmund T. An irreversible cohesive zone model for interface fatigue crack growth simulation. Eng Fract Mech 2003;70:209-32.

[17] Elices M, Guinea G, Gomez J, Planas J. The cohesive zone model: advantages, limitations and challenges. Eng Fract Mech 2002;69:137-63.

[18] Chandra N. Cohesive Zone Approach to Multiscale Modeling of Nanotube Reinforced Composites. Cohesive zone approach to multiscale modeling of nanotube reinforced composites 2007;FAMU-37099.

[19] Koo B, Subramanian N, Chattopadhyay A. Molecular Dynamics Study of Brittle Fracture in Epoxy-based Thermoset Polymer. Composites Part B: Engineering 2016:433-9. 
[20] Subramanian N, Koo B, Rai A, Chattopadhyay A. A Multiscale Damage Initiation Model for CNT-Enhanced Epoxy Polymers. 20 ${ }^{\text {th }}$ Int. Conf. on Composite Materials, Copenhagen 2015.

[21] Rai A, Subramanian N, Koo B, Chattopadhyay A. Multiscale damage analysis of carbon nanotube nanocomposite using a continuum damage mechanics approach. J Composite Mater 2016:0021998316654304.

[22] Rai A, Subramanian N, Chattopadhyay A. Investigation of piezo-resistivity in CNT nanocomposites under damage. 2016:980017,980017-11.

[23] Li C, Strachan A. Molecular dynamics predictions of thermal and mechanical properties of thermoset polymer EPON862/DETDA. Polymer 2011;52:2920-8.

[24] Subramanian N, Rai A, Chattopadhyay A. Atomistically informed stochastic multiscale model to predict the behavior of carbon nanotube-enhanced nanocomposites. Carbon 2015;94:661-72.

[25] Koo B, Liu Y, Zou J, Chattopadhyay A, Dai L. Study of glass transition temperature (Tg) of novel stress-sensitive composites using molecular dynamic simulation. Modell Simul Mater Sci Eng 2014;22:065018.

[26] Lu W, Wu J, Song J, Hwang K, Jiang L, Huang Y. A cohesive law for interfaces between multi-wall carbon nanotubes and polymers due to the van der Waals interactions. Comput Methods Appl Mech Eng 2008;197:3261-7.

[27] Tan H, Jiang L, Huang Y, Liu B, Hwang K. The effect of van der Waals-based interface cohesive law on carbon nanotube-reinforced composite materials. Composites Sci Technol 2007;67:2941-6.

[28] Plimpton S. Fast parallel algorithms for short-range molecular dynamics. Journal of computational physics 1995;117:1-19.

[29] Jorgensen WL, Maxwell DS, Tirado-Rives J. Development and testing of the OPLS allatom force field on conformational energetics and properties of organic liquids. J Am Chem Soc 1996;118:11225-36.

[30] Halgren TA. Merck molecular force field. I. Basis, form, scope, parameterization, and performance of MMFF94. Journal of computational chemistry 1996;17:490-519.

[31] Singh SK, Srinivasan SG, Neek-Amal M, Costamagna S, van Duin AC, Peeters F. Thermal properties of fluorinated graphene. Physical Review B 2013;87:104114.

[32] Johnston J, Koo B, Subramanian N, Chattopadhyay A. Modeling the Molecular Structure of the Carbon Fiber/Polymer Interphase in Composites for Multiscale Analysis . Composites Part B: Engineering 2016; (in press).

[33] García EJ, Hart AJ, Wardle BL. Long carbon nanotubes grown on the surface of fibers for hybrid composites. AIAA J 2008;46:1405-12. 
[34] Tvergaard V. Effect of fibre debonding in a whisker-reinforced metal. Materials science and engineering: A 1990;125:203-13.

[35] Lin G, Geubelle P, Sottos N. Simulation of fiber debonding with friction in a model composite pushout test. Int J Solids Structures 2001;38:8547-62.

[36] Li S, Ghosh S. Modeling interfacial debonding and matrix cracking in fiber reinforced composites by the extended Voronoi cell FEM. Finite Elements Anal Des 2007;43:397-410.

[37] Paggi M, Wriggers P. A nonlocal cohesive zone model for finite thickness interfaces-Part I: mathematical formulation and validation with molecular dynamics. Computational Materials Science 2011;50:1625-33.

[38] Tsai J, Patra A, Wetherhold R. Finite element simulation of shaped ductile fiber pullout using a mixed cohesive zone/friction interface model. Composites Part A: Applied Science and Manufacturing 2005;36:827-38. 


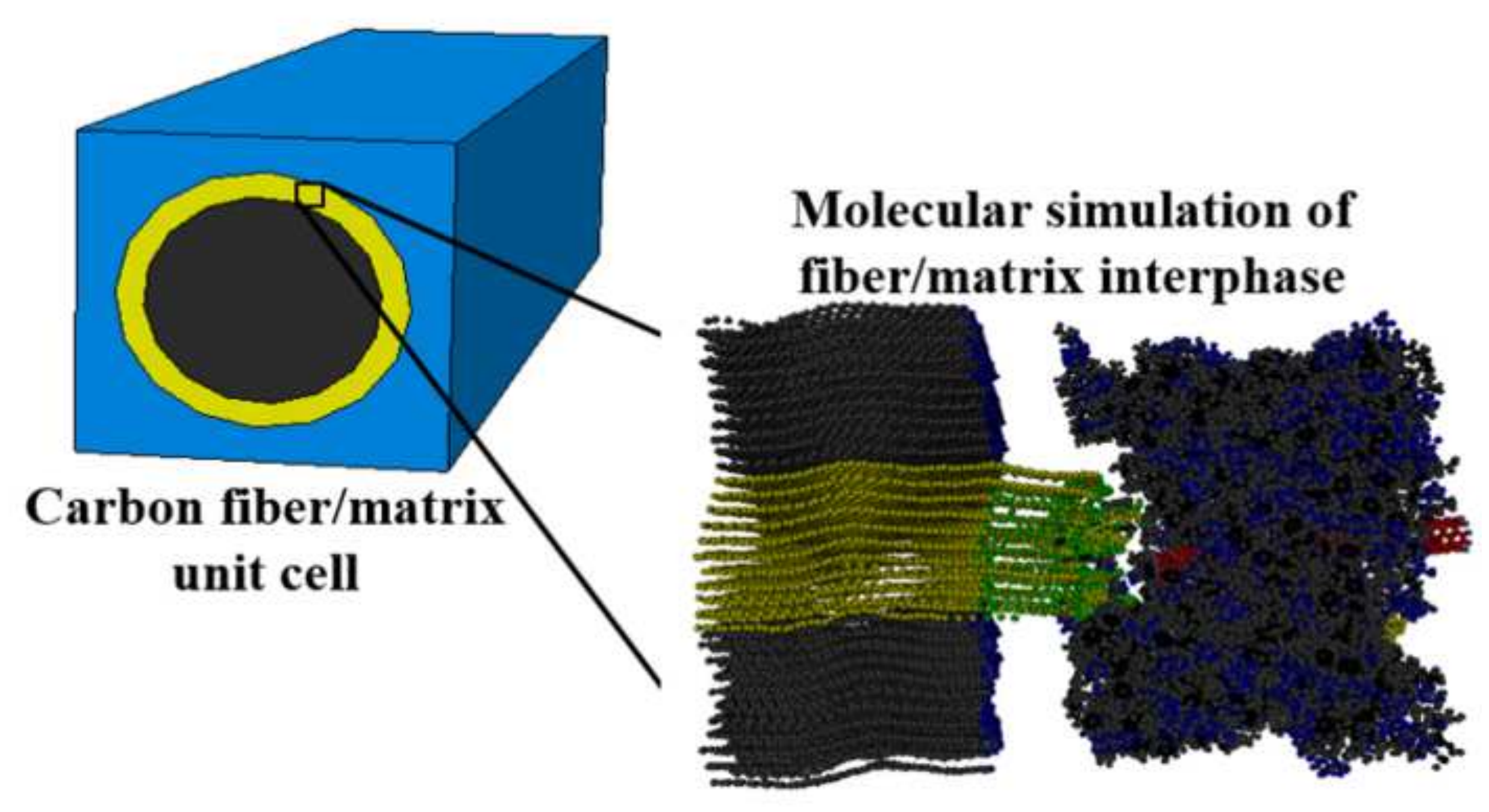

Molecular simulation of fiber/matrix interphase

Carbon fiber $/ \mathrm{m}$
unit cell

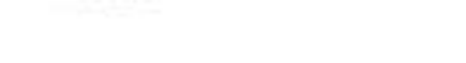

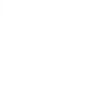

.

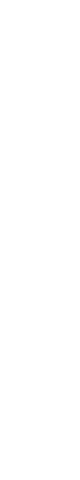

